\title{
Extreme fragmentation and complex kinematics at the center of the L1287 cloud
}

\author{
Carmen Juárez ${ }^{1,2,3}$, Hauyu Baobab Liu ${ }^{4}$, Josep M. Girart ${ }^{1,2}$, Aina Palau ${ }^{5}$, Gemma Busquet ${ }^{1,2}$, \\ Roberto Galván-Madrid ${ }^{5}$, Naomi Hirano ${ }^{6}$, and Yuxin $\operatorname{Lin}^{7,8}$ \\ ${ }^{1}$ Institut de Ciències de l'Espai, CSIC, Campus UAB, Carrer de Can Magrans, s/n 08193 Cerdanyola del Vallès, Barcelona, Catalonia, \\ Spain \\ ${ }^{2}$ Institut d'Estudis Espacials de Catalunya (IEEC), Barcelona, Catalonia, Spain \\ ${ }^{3}$ Departament de Física Quàntica i Astrofísica, Institut de Ciències del Cosmos (ICCUB) ${ }^{\star}$, Universitat de Barcelona (IEEC-UB), \\ Martí Franquès 1, 08028 Barcelona, Catalonia, Spain \\ ${ }^{4}$ European Southern Observatory (ESO), Karl-Schwarzschild-Str. 2, 85748 Garching, Germany \\ e-mail: hyliu@asiaa.sinica.edu.tw \\ 5 Instituto de Radioastronomía y Astrofísica, Universidad Nacional Autónoma de México, PO Box 3-72, 58090, Morelia, \\ Michoacán, Mexico \\ ${ }^{6}$ Institute of Astronomy and Astrophysics, Academia Sinica, PO Box 23-141, Taipei 106, Taiwan \\ ${ }^{7}$ National Astronomical Observatories, Chinese Academy of Sciences, Chaoyang, PR China \\ ${ }^{8}$ Max-Planck-Institut für Radioastronomie, 53121 Bonn, Germany
}

Received 2 September 2018 / Accepted 19 November 2018

\begin{abstract}
Aims. The filamentary $\sim 10$-pc-scale infrared dark cloud L1287 located at a parallax distance of $\sim 929 \mathrm{pc}$ is actively forming a dense cluster of low-mass young stellar objects (YSOs) at its inner $\sim 0.1 \mathrm{pc}$ region. To help understand the origin of this low-mass YSO cluster, the present work aims at resolving the gas structures and kinematics with high angular resolution.

Methods. We performed $\sim 1^{\prime \prime}$ angular resolution ( $\sim 930 \mathrm{AU}$ ) observations at $\sim 1.3 \mathrm{~mm}$ wavelengths using the Submillimeter Array (SMA), which simultaneously cover the dust continuum emission and various molecular line tracers for dense gas, warm gas, shocks, and outflows.

Results. From a 1.3-mm continuum image with a resolution of $\sim 2^{\prime \prime}$ we identified six dense cores, namely SMA1-6. Their gas masses are in the range of $\sim 0.4-4 M_{\odot}$. From a $1.3-\mathrm{mm}$ continuum image with a resolution of $\sim 1^{\prime \prime}$, we find a high fragmentation level, with 14 compact millimeter sources within 0.1 pc: SMA3 contains at least nine internal condensations; SMA5 and SMA6 are also resolved with two internal condensations. Intriguingly, one condensation in SMA3 and another in SMA5 appear associated with the known accretion outburst YSOs RNO 1C and RNO 1B. The dense gas tracer DCN (3-2) well traces the dust continuum emission and shows a clear velocity gradient along the NW-SE direction centered at SMA3. There is another velocity gradient with opposite direction around the most luminous YSO, IRAS $00338+6312$.

Conclusions. The fragmentation within $0.1 \mathrm{pc}$ in L1287 is very high compared to other regions at the same spatial scales. The incoherent motions of dense gas flows are sometimes interpreted by being influenced by (proto)stellar feedback (e.g., outflows), which is not yet ruled out in this particular target source. On the other hand, the velocities (with respect to the systemic velocity) traced by DCN are small, and the directions of the velocity gradients traced by DCN are approximately perpendicular to those of the dominant $\mathrm{CO}$ outflow(s). Therefore, we alternatively hypothesize that the velocity gradients revealed by DCN trace the convergence from the $\gtrsim 0.1 \mathrm{pc}$ scales infalling motion towards the rotational motions around the more compact $(\sim 0.02 \mathrm{pc})$ sources. This global molecular gas converging flow may feed the formation of the dense low-mass YSO cluster. Finally, we also found that IRAS $00338+6312$ is the most likely powering source of the dominant CO outflow. A compact blue-shifted outflow from RNO 1C is also identified.
\end{abstract}

Key words. stars: formation - ISM: individual objects: L1287 - ISM: molecules

\section{Introduction}

Molecular gas filaments collapsing towards the center of molecular clouds may pile up where the centrifugal force is becoming important compared with the gravitational force, and then form dense stellar clusters. The observationally very well resolved examples include the $L \sim 10^{7} L_{\odot}$ OB cluster-forming region W49A (e.g. Keto et al. 1991; Galván-Madrid et al. 2013; Lin et al. 2016), the $L \sim 10^{6} L_{\odot}$ OB cluster-forming region G10.6-0.4 (e.g. Keto et al. 1987; Liu et al. 2012a), the $L \sim 10^{5} L_{\odot}$ OB

^ The ICCUB is a CSIC-Associated Unit through the Institut de Ciències de l'Espai (ICE). cluster-forming region $\mathrm{G} 33.92+0.11$ (e.g. Liu et al. 2012b, 2015 ), and the $L \sim 10^{4} \quad L_{\odot} \quad$ OB cluster-forming region NGC6334 V (e.g. Juárez et al. 2017). Is it plausible to form dense clusters of low-mass young stellar objects (YSOs) with a similar mechanism (see Corsaro et al. 2017; Mapelli 2017)?

To try and answer this question, we study the filamentary dark molecular cloud, L1287, which is located at a distance of $929_{-33}^{+34} \mathrm{pc}$ (Rygl et al. 2010). Its inner, densest $\sim 0.1$-pc region is forming a $L \sim 10^{3} L_{\odot}$ low-mass YSO cluster (e.g. Estalella et al. 1993; Quanz et al. 2007). Four of the YSOs, namely VLA1-4, are known to be associated with thermal radio jets (Anglada et al. 1994). Based on the previous, lower angular-resolution 
Table 1. SMA observations summary.

\begin{tabular}{lccc}
\hline \hline Array configuration & Subcompact & Compact & Extended \\
\hline Observing date & 2014 Feb 10, July 25 & 2013 Aug 2 & 2013 Oct 24, 25 \\
$\tau_{225 \mathrm{GHz}}$ & $0.14,0.12$ & 0.13 & $0.25,0.16$ \\
Number of antennas & 6,7 & 5 & 6 \\
Time on target (h) & 2,5 & 8 & 8 \\
Flux calibrator & Callisto, Neptune & Uranus & Uranus \\
Passband calibrator & $0102+584$ & $3 \mathrm{c} 84$ & $3 \mathrm{c} 454.3$ \\
Gain calibrator & $0102+584$ & $0102+584$ & $0102+584$ \\
\hline
\end{tabular}

observations of $\mathrm{C}^{18} \mathrm{O}$ (1-0), Umemoto et al. (1999) estimated the enclosed gas mass over the $\sim 0.5$-pc-scale parent molecular gas structure to be $\sim 120 M_{\odot}$. Contrasting to the aforementioned OB cluster-forming regions, the bolometric luminosity of L1287 is not contributed by the nuclear burning of OB stars, but instead is contributed by the accretion luminosity of the low-mass protostars, including two known FU Orionis objects ${ }^{1}$ RNO 1B/1C (Staude \& Neckel 1991; Kenyon et al. 1993; McMuldroch et al. 1995; Quanz et al. 2007), and a $L \sim 600 L_{\odot}$ Class 0/I YSO IRAS $00338+6312$ (Anglada et al. 1994). Earlier observations resolved bipolar CO outflow(s) aligning in the NE-SW direction (Snell et al. 1990; Yang et al. 1991; Fehér et al. 2017), although the identification of the powering source(s) remains uncertain.

In this paper, we report the $\sim 1^{\prime \prime}(\sim 930$ AU)-angularresolution observations with the Submillimeter Array (SMA) ${ }^{2}$, covering the inner $\sim 0.25$-pc region of L1287. By examining the resolved $1.3 \mathrm{~mm}$ dust continuum emission and various molecular line tracers of dense gas, warm gas, shocks, and outflows, our aim is to elucidate how the gas flows converge from parsec scales (e.g., due to gravitational collapse) down to $\sim 1000 \mathrm{AU}$ scales, and how they are dynamically forming the low-mass YSO cluster. We also provide a more definite identification of the powering source of the previously reported molecular outflows.

The details of our observations are presented in Sect. 2. The observational results from the dust continuum and molecular line emission are given in Sect. 3. In Sect. 4 we present an analysis of fragmentation, dense gas kinematics, and powering sources of the previously reported $\mathrm{CO}$ outflows. Our conclusions are given in Sect. 5.

\section{Observations and data reduction}

\subsection{Submillimeter Array observations}

We performed five tracks of SMA observations at $\sim 1.3 \mathrm{~mm}$ wavelengths towards the inner $\sim 0.25-\mathrm{pc}$ region of $\mathrm{L} 1287$ between August 2013 and July 2014, which are summarized in Table 1. The pointing and phase referencing centers of the observations are $\mathrm{RA}(\mathrm{J} 2000)=00^{\mathrm{h}} 36^{\mathrm{m}} 46.65^{\mathrm{s}}, \operatorname{Dec}(\mathrm{J} 2000)=$ $+63^{\circ} 28^{\prime} 57.90^{\prime \prime}$. The projected baselines of these observations range from $\sim 4$ to $\sim 140 \mathrm{k} \lambda$. The system temperature $T_{\text {sys }}$ for all the observations was around $150 \mathrm{~K}$.

\footnotetext{
1 FU Orionis objects are young, pre-main sequence stars which are observed to increase their brightness by 4-6 mag in the optical and remain bright for decades (Herbig 1977). The large-amplitude flares are attributed to enhanced accretion from the surrounding circumstellar disk (Hartmann \& Kenyon 1985).

2 The Submillimeter Array is a joint project between the Smithsonian Astrophysical Observatory and the Academia Sinica Institute of Astronomy and Astrophysics, and is funded by the Smithsonian Institution and the Academia Sinica (Ho et al. 2004).
}

The observations were obtained with the $230 \mathrm{GHz}$ receiver, with $4 \mathrm{GHz}$ bandwidth in each of the upper and lower sidebands. The correlator consisted of 48 chunks, each with a bandwidth of $104 \mathrm{MHz}$. The frequency was centered at $231.3 \mathrm{GHz}$ at chunk 35 of the upper sideband (USB). This configured the correlator with the lower sideband (LSB) covering from 216.48 to $220.46 \mathrm{GHz}$ and the USB from 228.46 to $232.43 \mathrm{GHz}$. These frequency ranges covered the molecular lines listed in Table A.1. The ${ }^{13} \mathrm{CO}$ and $\mathrm{N}_{2} \mathrm{D}^{+}$lines were observed with 512 channels per chunk and a spectral resolution of $0.26 \mathrm{~km} \mathrm{~s}^{-1}$, and the line $\mathrm{NH}_{2} \mathrm{D}$ was observed with 256 channels per chunk giving $0.53 \mathrm{~km} \mathrm{~s}^{-1}$ spectral resolution. The remaining lines were observed with 128 channels per chunk and a $1.06 \mathrm{~km} \mathrm{~s}^{-1}$ spectral resolution. To generate the $1.3 \mathrm{~mm}$ continuum, we averaged the line-free channels in the lower and upper sidebands. The continuum data have been reported by Liu et al. (2018) as part of a SMA survey towards 29 accretion-outburst YSOs. These latter authors did not analyze the details of the extended structures, and did not analyze spectral line data.

The calibration for absolute flux, bandpass, and gain were carried out using the MIR IDL software package ${ }^{3}$. The images were created using the Multichannel Image Reconstruction, Image Analysis, and Display (MIRIAD, Sault et al. 1995) software package.

To map the rather extended gas flows, we used ROBUST $=1$ weighting (Briggs 1995) to create the 1.3-mm dust continuum emission map using all data listed in Table 1, which yielded a $2^{\prime \prime} 28 \times 2^{\prime \prime} 17\left(\mathrm{PA}=-19^{\circ}\right)$ synthesized beam, and an rms noise level of $0.6 \mathrm{mJy}^{\text {beam }}{ }^{-1}$. In addition, we created a higher angular-resolution 1.3-mm dust continuum emission map of spatially compact sources using only the observations taken in the extended array configuration. We used a ROBUST $=0$ weighting, which yielded a synthesized beam of $0^{\prime \prime} 96 \times 0^{\prime \prime} 79\left(\mathrm{PA}=-22^{\circ}\right)$ and an RMS noise level of $0.5 \mathrm{mJy}^{\text {beam }}{ }^{-1}$.

To present the maps of the molecular lines, we applied three different weightings. For most of the lines that show extended emission we used a ROBUST $=2$ weighting (i.e., natural weighting), which provides the best sensitivity. For the CO (2-1) line we used a ROBUST $=0$ weighting to obtain a better angular resolution. Finally, for OCS (18-17), HNCO 10(0,10)-9(0,9), and $\mathrm{CH}_{3} \mathrm{OH} 3(-2,2)-4(-1,4)$, which present spatially compact emission, we used only data from the extended and compact array configurations and a ROBUST $=2$ weighting.

\subsection{Archival Herschel images}

To present the structures of the parent molecular cloud on scales of 1-10 pc, and to estimate the dust temperature, we retrieved the Herschel-SPIRE 250, 350, and $500 \mu \mathrm{m}$ images, which were

3 https://www.cfa.harvard.edu/ cqi/mircook.html 

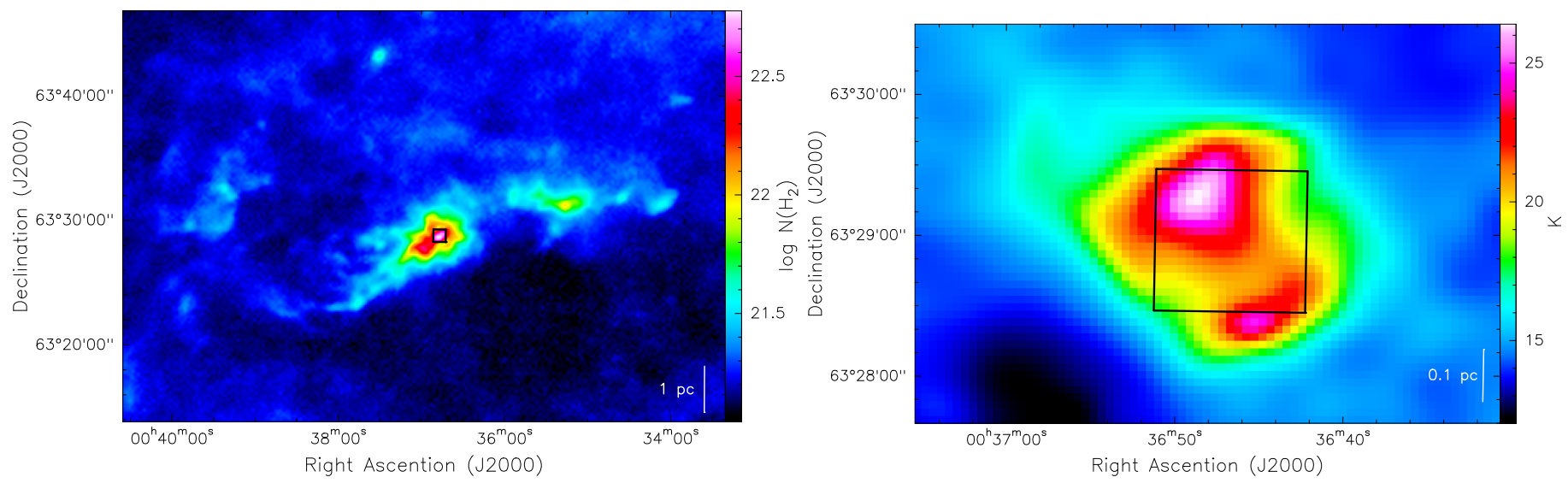

Fig. 1. 37" angular resolution gas column density $\left(N\left(\mathrm{H}_{2}\right)\right.$; left panel) and dust temperature $\left(T_{d}\right.$; right panel) maps of L1287 (for more details see Sect. 2.2). The black box in both panels indicates the field of view of the top panel of Fig. 2, which presents the 1.3 mm continuum image taken with the SMA.

taken as part of the Herschel infrared Galactic Plane (Hi-GAL) survey (obsID:1342249229, Molinari et al. 2010).

We smoothed the 250 and $350 \mu \mathrm{m}$ images to the angular resolution of the $500 \mu \mathrm{m}$ image $\left(37^{\prime \prime}\right)$, and then derived gas column density $\left(N\left(\mathrm{H}_{2}\right)\right)$ and dust temperature $\left(T_{d}\right)$ pixel-by-pixel by fitting the modified-black-body spectrum

$S_{v}=\Omega_{m} B_{v}\left(T_{d}\right)\left(1-e^{-\tau_{v}}\right)$,

where $S_{v}$ is the flux observed at frequency $v, \Omega_{m}$ is the considered solid angle, $B_{v}\left(T_{d}\right)$ is the Planck function at $T_{d}$, and $\tau_{v}$ is the dust optical depth. We converted $\tau_{v}$ to the gas column density $N\left(\mathrm{H}_{2}\right)$ by assuming

$N\left(\mathrm{H}_{2}\right)=R \frac{\tau_{v}}{\kappa_{v} \mu m_{\mathrm{H}}}$,

where $R$ is the gas-to-dust mass ratio which we assumed to be $100, \mu=2.8$ is the mean molecular weight, $\mathrm{m}_{\mathrm{H}}$ is the mass of a hydrogen atom, and $\kappa_{v}$ is the dust mass opacity which is assumed to have the following dependence on frequency $v$

$\kappa_{v}=\kappa_{230 \mathrm{GHz}}\left[\frac{v[\mathrm{GHz}]}{230}\right]^{\beta}$,

where $\kappa_{230}=0.9 \mathrm{~cm}^{2} \mathrm{~g}^{-1}$ (Ossenkopf \& Henning 1994). We assumed a dust emissivity index $\beta$ of 1.8 . We refer to Hildebrand (1983) for an introduction to the formulation we are based on.

\section{Results}

\subsection{Dust continuum}

Figure 1 shows the large-scale $N\left(\mathrm{H}_{2}\right)$ and $T_{d}$ maps of L1287. On 1-10 pc scales this molecular cloud presents a filamentary morphology, with a dense molecular gas hub (see Myers 2009) located at its central $\sim 0.5 \mathrm{pc}$ region. The $T_{d}$ map shows two higher temperature lobes to the NE and SW of the hub, which may be associated with heated cavities created by the bipolar $\mathrm{CO}$ outflow(s) emanated from the embedded low-mass YSO cluster (Snell et al. 1990; Yang et al. 1991; Quanz et al. 2007).

Figure 2 shows the SMA $1.3 \mathrm{~mm}$ continuum image on the central region of the hub. The continuum emission at $1.3 \mathrm{~mm}$ can arise from dust thermal emission and free-free emission.
Based on the deep VLA observations of the centimeter free-free continuum emission presented in Anglada et al. (1994), we constrained the $1.3-\mathrm{mm}$ free-free emission to be $<0.5 \mathrm{mJy}$ towards IRAS $0038+6312$ and RNO 1C. When compared to the detected emission level in Fig. 2, we consider that the contribution of free-free emission is in general negligible.

The $2^{\prime \prime}$ resolution $1.3-\mathrm{mm}$ dust continuum emission (top panel of Fig. 2) splits up into six main cores (defined with at least a $35 \sigma$ closed contour), which are spatially separated by a mean distance of $\sim 0.03 \mathrm{pc}(\sim 6500 \mathrm{au})$, and have a mean size of $\sim 0.02 \mathrm{pc}(\sim 4500 \mathrm{au})$. Assuming that the dust emission is optically thin, we estimated the gas and dust masses of these cores based on the following formulation

$M=R \frac{d^{2} S_{v}}{B_{v}\left(T_{d}\right) \kappa_{v}}$,

where $d$ is the distance. Here we adopted $\kappa_{v}=0.9 \mathrm{~cm}^{2} \mathrm{~g}^{-1}$ for thin ice mantles after $10^{5} \mathrm{yr}$ of coagulation at a gas density of $10^{6} \mathrm{~cm}^{-3}$ and for the frequency of our observations (Ossenkopf \& Henning 1994). For these estimates we assumed $T_{d}=22 \mathrm{~K}$, which is the averaged dust temperature we derived in this region based on the Herschel data (Fig. 1, right).

The derived masses of these six main cores are in the range of 0.4-4 $M_{\odot}$. The central core (SMA3; harboring RNO 1C and VLA 1) and the northeastern core (SMA1; harboring IRAS $0038+6312$ and VLA 3) are the most massive ones, with $\sim 4$ and $\sim 2 M_{\odot}$, respectively. The faintest core, SMA4, which may harbor VLA 4, is located SE of the central core, and has a mass of $\sim 0.4 M_{\odot}$. The faint and small core (SMA5) located S of the central core seems to be associated with the FU Orionis object RNO $1 \mathrm{~B}$ and has a mass of $\sim 0.6 M_{\odot}$. The overall mass recovered by our SMA $1.3-\mathrm{mm}$ continuum image is $22 M_{\odot}$.

To better resolve the internal fragmentation in this region, we present the $1.3-\mathrm{mm}$ continuum image generated using only the extended configuration data (i.e., $\sim 1^{\prime \prime}$ angular resolution; Table 1), which is shown in the bottom panel of Fig. 2. The highest-mass core, SMA3, is resolved into a $\sim 0.02$ pc scale clumpy toroid containing at least nine internal gas condensations; SMA3b and SMA3c may be associated with the $\mathrm{YSO}(\mathrm{s})$ RNO 1C and VLA 1; SMA2 appears to be a $\sim 0.02$ pc scale, elongated arm-like structure connecting to the clumpy toroid from the north; SMA5 and SMA6 may be parts of another $\sim 0.04$-pc-scale, elongated arm-like structure connecting to the 

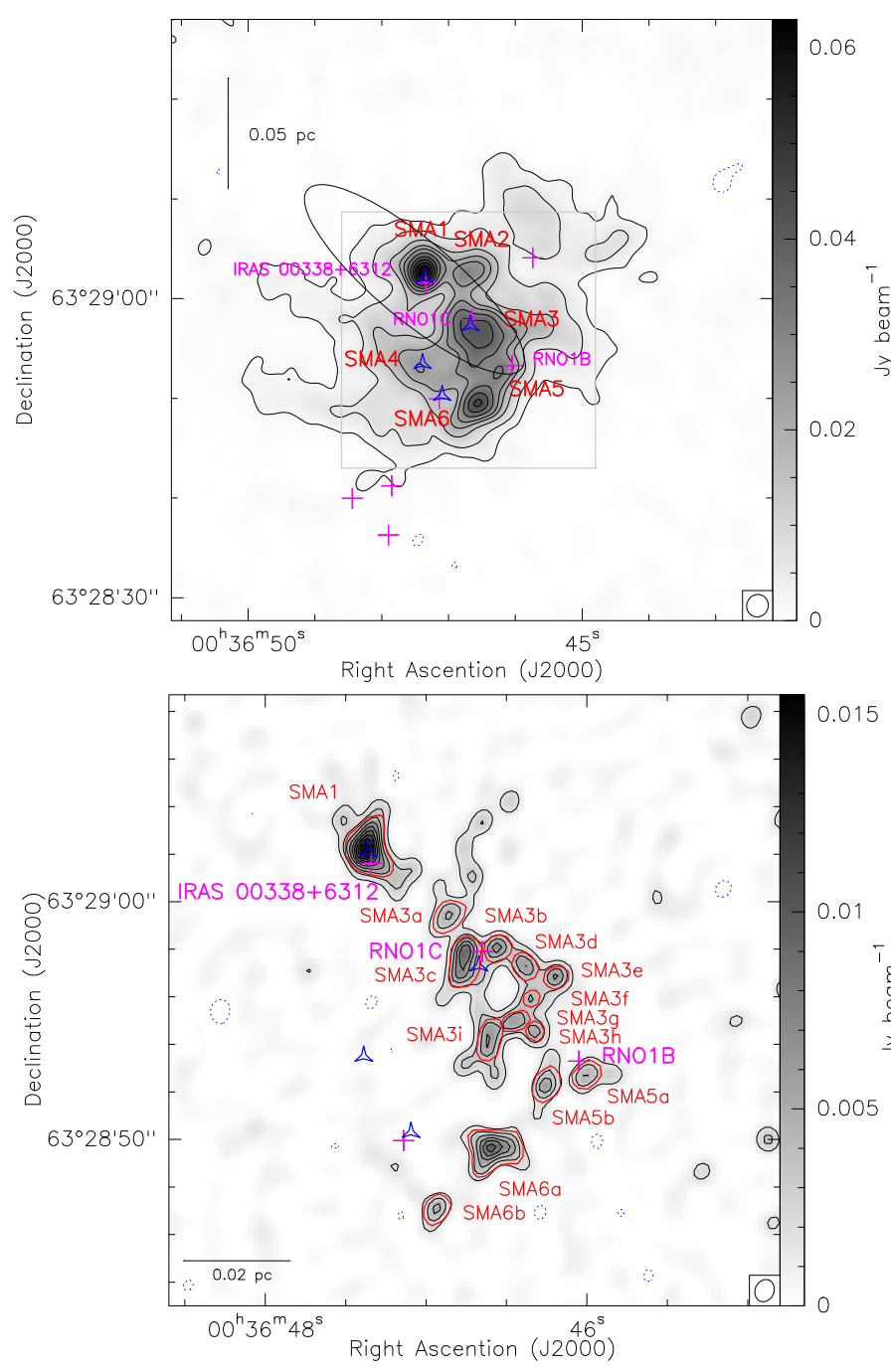

Fig. 2. Top panel: 1.3-mm dust continuum emission with extended, compact, and subcompact configurations. Contours are $-6,-3,3,6$, $9,20,30,38,49,60,70,80$, and 90 times the rms level of the map, $0.6 \mathrm{mJy}$ beam $^{-1}$. Black ellipse corresponds to the position error ellipse for IRAS $00338+6312$. The synthesized beam located at the bottom right is $2^{\prime \prime} 28 \times 2^{\prime \prime} 17, \mathrm{PA}=-19^{\circ}$. The gray square corresponds to the field of view of the bottom panel. A scale bar is located in the upper-left corner. Bottom panel: 1.3-mm dust continuum emission with extended configuration. Contours are $-3,3,6,9,12,15,18,21,24,27$ and 30 times the rms level of the map, $0.5 \mathrm{mJy}$ beam $^{-1}$. Sources (labeled in red) are defined as having at least a $7 \sigma$ closed contour. Red contours are drawn following the 6 and 9 sigma contour levels for the most extended sources. The synthesized beam located at the bottom right is $0^{\prime \prime} 96 \times 0^{\prime \prime} 79, \mathrm{PA}=-22^{\circ}$. A scale bar is shown in the lower left corner. Blue concave hexagons and pink crosses in both panels are radio (Anglada et al. 1994) and infrared (Quanz et al. 2007) sources, respectively.

clumpy toroid from the south. Overall, we identify 14 condensations with at least a $7 \sigma$ closed contour. We measured the flux density of each condensation by fitting an elliptical Gaussian using the MIRIAD task IMFIT, and then based on Eq. (4) we estimated the gas and dust masses. The polygons for fitting the Gaussian were drawn following the $6 \sigma$ and the $9 \sigma$ contour levels for the most extended sources $\left(1 \sigma=0.5 \mathrm{mJy} \mathrm{beam}^{-1}\right)$. The derived masses range between $\sim 0.1$ and $\sim 0.7 M_{\odot}$. The properties derived from these fits also included the peak position, the deconvolved angular size and position angle, and the intensity of the peak. The results are listed in Table 2. We note that the total flux obtained taking into account only the extended configuration is $\sim 16 \%$ of the total flux obtained with all the configurations combined. This is due to the filtering effect of the extended emission.

\subsection{Molecular lines}

In addition to the dust continuum emission, a number of molecular lines were detected with the SMA within the $8 \mathrm{GHz}$ bandwidth. We identified 15 molecular lines of $\mathrm{CH}_{3} \mathrm{OH}, \mathrm{SO}$, OCS, $\mathrm{SiO}, \mathrm{H}_{2} \mathrm{CO}, \mathrm{HNCO}, \mathrm{DCN}, \mathrm{DNC}$ and CO. Table A.1 lists the frequency, the energy of the upper level, and the estimated critical density for each transition. In Fig. 3, we present the velocity integrated emission (i.e., moment 0 ) maps of several of the detected transitions in L1287.

The shock tracer $\mathrm{SiO}$ (5-4) reveals compact emission towards IRAS $00338+6312$, RNO1C, VLA 4 and the dust continuum core SMA6. Also, it presents compact emission, neither associated with dust continuum emission nor to any YSO, towards the east, west, and southwestern parts of the region. SO 6(5)-5(4) emission traces similar structures with those traced by $\mathrm{SiO}(5-4)$, but presents more prominent extended emission covering the central part of the observed region. In addition, the SO 6(5)-5(4) emission is more extended than $\mathrm{SiO}$ emission in the southwest, forming a "V" shaped structure. $\mathrm{CH}_{3} \mathrm{OH} 8(-1,8)$ $7(0,7), \mathrm{H}_{2} \mathrm{CO} 3(0,3)-2(0,2)$, and 3(2,1)-2(2,0) emission present similar morphology to $\mathrm{SiO}$ and $\mathrm{SO}$, but additionally show bright emission towards VLA 2 and are fainter around RNO 1C. DCN (3-2) and $\mathrm{H}_{2} \mathrm{CO} 3(2,1)-2(2,0)$ transitions trace well the dust continuum emission. The emission extends over the dust continuum presenting the strongest emission at IRAS $0038+6312$. In addition, DCN (3-2) shows strong emission towards the toroidal dust structure, with fainter emission coinciding with RNO $1 \mathrm{C}$ and the center of the dust continuum toroid. Moreover, unresolved emission of $\mathrm{CH}_{3} \mathrm{OH} 5(1,4)-4(2,2), \mathrm{CH}_{3} \mathrm{OH} 3(-2,2)-$ $4(-1,4)$, OCS $(18-17)$ and HNCO $10(0,10)-9(0,9)$ is detected at IRAS $0038+6312$.

In Fig. 4 we present the intensity-weighted averaged velocity (i.e., moment 1) maps of the lines presented in Fig. 3. A Gaussian fit to the DCN (3-2) spectrum averaged from the entire observed region indicates that the systemic velocity of this system is $-17.4 \mathrm{~km} \mathrm{~s}^{-1}$. The dense core tracers $\mathrm{H}_{2} \mathrm{CO} 3(0,3)$ $2(0,2)$ and $3(2,1)-2(2,0)$, SO 6(5)-5(4) and more clearly DCN (3-2) show a NW-SE large-scale $(\sim 0.1 \mathrm{pc})$ velocity gradient between -15 and $-19 \mathrm{~km} \mathrm{~s}^{-1}$ centered at the dust central core (SMA3) near RNO1C and VLA 1. We note that previous singledish observations of $\mathrm{H}^{13} \mathrm{CO}^{+}(1-0)$ also resolved a velocity gradient in the same direction, but extended to a much larger angular scale $\left(\sim 2^{\prime}, \sim 0.6 \mathrm{pc}\right.$; see Umemoto et al. 1999). In addition, on a smaller spatial scale and around IRAS $0338+6312$, these molecular lines also reveal a velocity gradient in the reversed direction. These velocity gradients are perpendicular to the previously reported, NE-SW bipolar outflow (Snell et al. 1990; Yang et al. 1991). The velocity gradient around IRAS $0338+6312$ is also seen in $\mathrm{CH}_{3} \mathrm{OH} 8(-1,8)-7(0,7)$. The emission towards the SW of the dust continuum emission seen in $\mathrm{H}_{2} \mathrm{CO}, \mathrm{SO}$, and $\mathrm{SiO}$ is clearly blue-shifted with a velocity of approximately $-24 \mathrm{~km} \mathrm{~s}^{-1}$.

To study the kinematics in the region in more detail, we present the blue- and red-shifted emission of DCN (3-2) in Fig. 5. The first panel presents the emission at $\pm 1.2 \mathrm{~km} \mathrm{~s}^{-1}$ from the systemic velocity. The blue- and red-shifted emission shows two structures forming the large-scale and reversed small-scale velocity gradients. At higher velocities (at \pm 2.4 and 
Table 2. Parameters of the sources detected with the SMA at $1.3 \mathrm{~mm}$ dust continuum emission.

\begin{tabular}{|c|c|c|c|c|c|c|c|c|}
\hline \multirow[b]{2}{*}{ Source } & \multicolumn{2}{|c|}{ Position $^{a}$} & \multirow{2}{*}{$\begin{array}{c}\text { Deconv. ang. size }{ }^{a} \\
\left({ }^{\prime \prime} \times{ }^{\prime \prime}\right)\end{array}$} & \multirow{2}{*}{$\begin{array}{l}\mathrm{PA}^{a} \\
\left({ }^{\circ}\right)\end{array}$} & \multirow{2}{*}{$\begin{array}{c}I_{v}^{\text {peak }_{a}} \\
\left(\mathrm{mJy} \mathrm{beam}^{-1}\right)\end{array}$} & \multirow{2}{*}{$\begin{array}{c}S_{v}{ }^{b} \\
(\mathrm{mJy})\end{array}$} & \multirow{2}{*}{$\begin{array}{l}\operatorname{Mass}^{c} \\
\left(M_{\odot}\right)\end{array}$} & \multirow[b]{2}{*}{ Association $^{d}$} \\
\hline & $\alpha(\mathrm{J} 2000)$ & $\delta(\mathrm{J} 2000)$ & & & & & & \\
\hline SMA1 & $00: 36: 47.366$ & $63: 29: 02.237$ & $1.6 \pm 0.3 \times 0.9 \pm 0.2$ & $8 \pm 15$ & $13.84 \pm 1.72$ & $41.4 \pm 8.3$ & 0.69 & CM:VLA 3/IR:IRAS \\
\hline SMA3a & $00: 36: 46.860$ & $63: 28: 59.414$ & $1.2 \pm 0.2 \times 0.6 \pm 0.1$ & $-44 \pm 8$ & $4.71 \pm 0.28$ & $9.2 \pm 1.9$ & 0.15 & - \\
\hline SMA3b & $00: 36: 46.562$ & $63: 28: 58.040$ & $0.9 \pm 0.3 \times 0.6 \pm 0.2$ & $-69 \pm 31$ & $6.45 \pm 0.66$ & $11.1 \pm 2.3$ & 0.19 & IR:RNO1C \\
\hline SMA3c & $00: 36: 46.761$ & $63: 28: 57.583$ & $1.9 \pm 0.4 \times 0.6 \pm 0.1$ & $-9 \pm 6$ & $8.72 \pm 0.85$ & $24.7 \pm 5.0$ & 0.41 & CM:VLA 1 \\
\hline SMA3d & $00: 36: 46.387$ & $63: 28: 57.326$ & Point source & - & $5.93 \pm 0.24$ & $11.0 \pm 2.2$ & 0.18 & - \\
\hline SMA3e & 00:36:46.198 & $63: 28: 56.829$ & $0.6 \pm 0.1 \times 0.5 \pm 0.1$ & $-77 \pm 29$ & $6.24 \pm 0.35$ & $8.6 \pm 1.8$ & 0.14 & - \\
\hline SMA3f & $00: 36: 46.348$ & $63: 28: 55.930$ & $1.0 \pm 0.3 \times 0.6 \pm 0.1$ & $-25 \pm 11$ & $4.74 \pm 0.10$ & $8.6 \pm 1.8$ & 0.14 & - \\
\hline SMA3g & $00: 36: 46.450$ & 63:28:54.966 & $1.5 \pm 0.3 \times 0.4 \pm 0.2$ & $-70 \pm 10$ & $5.72 \pm 0.36$ & $13.0 \pm 2.7$ & 0.22 & - \\
\hline SMA3h & $00: 36: 46.330$ & $63: 28: 54.583$ & Point source & - & $5.41 \pm 0.39$ & $7.3 \pm 1.5$ & 0.12 & - \\
\hline SMA3i & $00: 36: 46.606$ & $63: 28: 54.248$ & $2.0 \pm 0.6 \times 0.6 \pm 0.2$ & $-13 \pm 8$ & $6.22 \pm 0.58$ & $18.4 \pm 3.8$ & 0.31 & - \\
\hline SMA5a & $00: 36: 46.000$ & $63: 28: 52.704$ & $1.1 \pm 0.3 \times 0.5 \pm 0.2$ & $-64 \pm 17$ & $4.50 \pm 0.42$ & $8.3 \pm 1.7$ & 0.14 & IR:RNO1B \\
\hline SMA5b & $00: 36: 46.255$ & $63: 28: 52.307$ & $0.9 \pm 0.2 \times 0.1 \pm 0.1$ & $-16 \pm 8$ & $5.63 \pm 0.46$ & $7.6 \pm 1.6$ & 0.13 & - \\
\hline SMA6a & $00: 36: 46.584$ & $63: 28: 49.613$ & $1.5 \pm 0.3 \times 0.9 \pm 0.2$ & $-82 \pm 22$ & $8.50 \pm 0.11$ & $25.7 \pm 5.2$ & 0.43 & - \\
\hline SMA6b & $00: 36: 46.936$ & $63: 28: 47.078$ & $0.7 \pm 0.1 \times 0.2 \pm 0.1$ & $-28 \pm 8$ & $4.96 \pm 0.24$ & $6.5 \pm 1.3$ & 0.11 & - \\
\hline
\end{tabular}

Notes. ${ }^{(a)}$ Position, deconvolved size, position angle (PA), peak intensity, flux density and the respective uncertainties derived from fitting a 2D Gaussian to each source using IMFIT task from MIRIAD. ${ }^{(b)}$ Error in flux density has been calculated as $\sqrt{\left(\sigma \theta_{\text {source }} / \theta_{\text {beam }}\right)^{2}+\left(\sigma_{\text {flux-scale }}\right)^{2}}($ Beltrán et al. 2001; Palau et al. 2013), where $\sigma$ is the rms of the map, $\theta_{\text {source }}$ and $\theta_{\text {beam }}$ are the size of the source and the beam, respectively, and $\sigma_{\text {flux-scale }}$ is the error in the flux scale, which takes into account the uncertainty on the calibration applied to the flux density of the source $\left(S_{v} \times \%_{\text {uncertainty }}\right)$ which we assumed to be $20 \% .{ }^{(c)}$ Masses derived assuming a dust temperature of $22 \mathrm{~K}$, and a dust (+gas) mass opacity coefficient at $1.3 \mathrm{~mm}$ of $0.9 \mathrm{~cm}^{2} \mathrm{~g}^{-1}$ (for thin ice mantles after $10^{5} \mathrm{yr}$ of coagulation at a gas density of $10^{6} \mathrm{~cm}^{-3}$, Ossenkopf \& Henning 1994). The uncertainty in the masses due to the opacity law and temperature is estimated to be a factor of $4 .{ }^{(d)}$ Association with signposts of stellar activity: IR = infrared source; $\mathrm{CM}=$ Centimeter radio source. IRAS refers to IRAS $0038+6312$.

$\pm 3.6 \mathrm{~km} \mathrm{~s}^{-1}$ ), the small-scale velocity gradient centered on IRAS $00338+6312$ becomes more compact.

Molecular outflow. In Fig. 6, we present the red- and blueshifted high-velocity emission of the $\mathrm{CO}(2-1)$ in different velocity ranges. At these high velocities, the $\mathrm{CO}(2-1)$ traces molecular outflows. In agreement with the previous observations by Snell et al. (1990) and Yang et al. (1991), the emission shows an extended NE-SW bipolar molecular outflow centered near IRAS $0038+6312$ (and VLA 3) and the FU Orionis object RNO 1C. However, IRAS $0038+6312$ seems to fall closer to the center of the bipolar structure (see first and second panels of Fig. 6). Moreover, the red- and blue-shifted emission in the NE and SW lobes, respectively, present clear cavity features, with the southern side of the blue-shifted lobe coinciding with the emission detected with the dense gas tracer $\mathrm{H}_{2} \mathrm{CO}$ and the shock tracers $\mathrm{SO}$ and $\mathrm{SiO}$. This strongly suggests that the outflow is dragging dense gas from this region (see Figs. 4 and 7), as already observed in other regions (e.g. HH 2: Girart et al. 2005; Lefloch et al. 2005). In the last two panels, at the highest velocities, the blue-shifted emission appears at the position of RNO1C with an elongated structure in the N-S direction.

To analyze only the highest-velocity gas from the CO (2-1) emission, the upper panel of Fig. 8 shows the integrated emission between -51 and $-47 \mathrm{~km} \mathrm{~s}^{-1}$. The emission at these velocities is compact and appears only very close to the position of RNO 1C. It is also important to note that the shock tracer $\mathrm{SiO}(5-4)$ shows emission towards both IRAS $0038+6312$ and RNO 1C (see Fig. 3).

\section{Discussion}

\subsection{Fragmentation in $L 1287$}

In Sect. 3.1 we presented the continuum emission at $1^{\prime \prime}$ angular resolution for L1287, which reveals 14 compact millimeter sources within a region of $0.1 \mathrm{pc}$. Given the rms noise of the $1^{\prime \prime}$ image, our mass sensitivity is $\sim 0.05 M_{\odot}$ (making the same assumptions as in Sect. 3.1). This mass sensitivity and our spatial resolution of $930 \mathrm{AU}$ are well below the Jeans mass $\left(\sim 0.6 M_{\odot}\right)$ and Jeans length $(\sim 6200 \mathrm{AU})$ for a region of density $10^{6} \mathrm{~cm}^{-3}$ and temperature of $20 \mathrm{~K}$ (following Palau et al. 2015), and are fully comparable to those reported by Palau et al. (2014, 2015). Thus, the SMA observations presented here are well suited to study fragmentation in L1287.

Palau et al. (2014, 2015) study the fragmentation level of a sample of 19 intermediate- and high-mass dense cores also within a spatial scale of $0.1 \mathrm{pc}$, and find fragmentation levels ranging from 1 up to 11 sources. The fragmentation level is defined by Palau et al. $(2014,2015)$ as the number of compact millimeter sources (with at least one closed contour) above 6 times the rms noise of the image, within a region of $0.1 \mathrm{pc}$ of diameter. Therefore, L1287, with 14 compact millimeter sources within $0.1 \mathrm{pc}$, presents a very high fragmentation level compared to the regions of this sample.

Palau et al. $(2014,2015)$ study the relation between the fragmentation level and different properties of the host cores, and find possible trends of fragmentation level increasing with the average density within $0.1 \mathrm{pc}$ (with a correlation coefficient of 0.89 ) and with the ratio of rotational to gravitational energy (with a correlation coefficient of 0.57 ), usually referred to as $\beta_{\text {rot }}$. Here, we therefore study whether the average density within $0.1 \mathrm{pc}$ and/or the rotation motions might explain the high fragmentation level in L1287.

In order to obtain a first approximation of the average density within $0.1 \mathrm{pc}$, we measured the flux density within an angular diameter of $22^{\prime \prime}$ (corresponding to $0.1 \mathrm{pc}$ ) in an image of the dust emission obtained with a single dish, so that we avoid the filtering of large-scale emission produced by interferometers. We chose to use the James Clerk Maxwell Telescope data at $450 \mu \mathrm{m}$ from Di Francesco et al. (2008) because this provides a main 

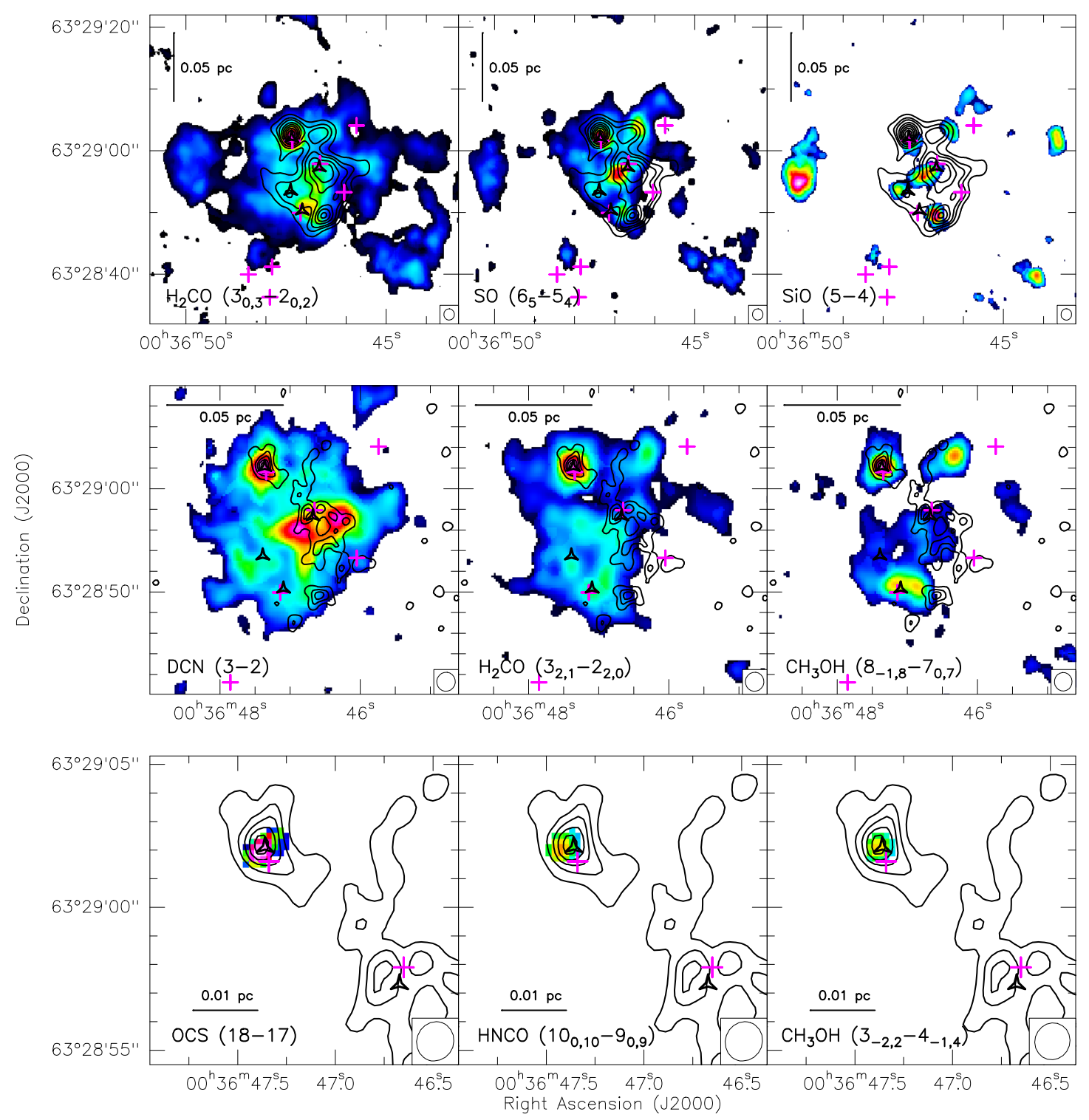

Fig. 3. Color scale image of the integrated intensity (i.e., moment 0) maps of selected molecular line tracers overlapped with the contour map of the $1.3 \mathrm{~mm}$ dust continuum emission (only using the extended configuration for the dust continuum emission in the middle and lower panels). Pink crosses and black triangles indicate infrared (Quanz et al. 2007) and radio (Anglada et al. 1994) sources, respectively. The synthesized beam of each molecule is located at the bottom-right corner.

beam of $11^{\prime \prime}$ (and an effective beam of 17.3"), which allows us to slightly resolve the emission within $0.1 \mathrm{pc}$. We obtained a flux density of $56 \mathrm{Jy}$ within $0.1 \mathrm{pc}$ for L1287. Taking into account an average dust temperature within $0.1 \mathrm{pc}$ of $20 \mathrm{~K}$ (from the Herschel data presented in Sect. 2.2), this corresponds to an average density of around $10^{6} \mathrm{~cm}^{-3}$. On the other hand, using the DCN velocity gradient of Fig. 10 (bottom), we estimated $\beta_{\text {rot }}$ for L1287, which is of 0.067 . In Fig. 9 we plot the fragmentation level versus average density within $0.1 \mathrm{pc}$, and also versus $\beta_{\text {rot }}$ for the sample of Palau et al. (2014, 2015), including the new results for L1287 (marked as a red symbol). These plots show that L1287 presents a relatively high density and a high $\beta_{\text {rot }}$ when compared to the entire sample of 19 massive dense cores of Palau et al. (2014, 2015). It therefore seems that both gravity and rotational energy could play an important role in the fragmentation process within L1287.

Considering the compact nature of the millimeter fragments, it seems reasonable to think that these fragments will form stars in the near future or are already associated with low-mass YSOs.
Thus, our data seem to indicate that L1287 is forming a dense cluster of deeply embedded low-mass YSOs.

\subsection{Dense gas kinematics}

For complicated cluster-forming regions resolved with $\mathrm{CO}$ outflows, it is always possible to attribute incoherent motions to the influence of outflow feedback or turbulence motions. While with the data presented here we cannot rule out these cases, below we discuss one possibility to coherently interpret the observed dense gas kinematics from large to small spatial scales as gravitational contraction.

An inverse P Cygni line profile (Pirogov et al. 2016), which is consistent with infalling motions on scales of $\gtrsim 0.1 \mathrm{pc}$, has been found towards $\mathrm{L} 1287$ using single-dish observations of $\mathrm{HCO}^{+}$ and $\mathrm{H}^{13} \mathrm{CO}^{+}$(1-0). On slightly larger scales, of $\sim 0.3-0.5 \mathrm{pc}$, Umemoto et al. (1999) resolved a blueshifted motion in the NW, and a redshifted motion in the SE. On $\sim 0.1-p c$ scales, our DCN (3-2) and $\mathrm{H}_{2} \mathrm{CO} 3(0,3)-2(0,2)$ line images (Fig. 4) trace a 

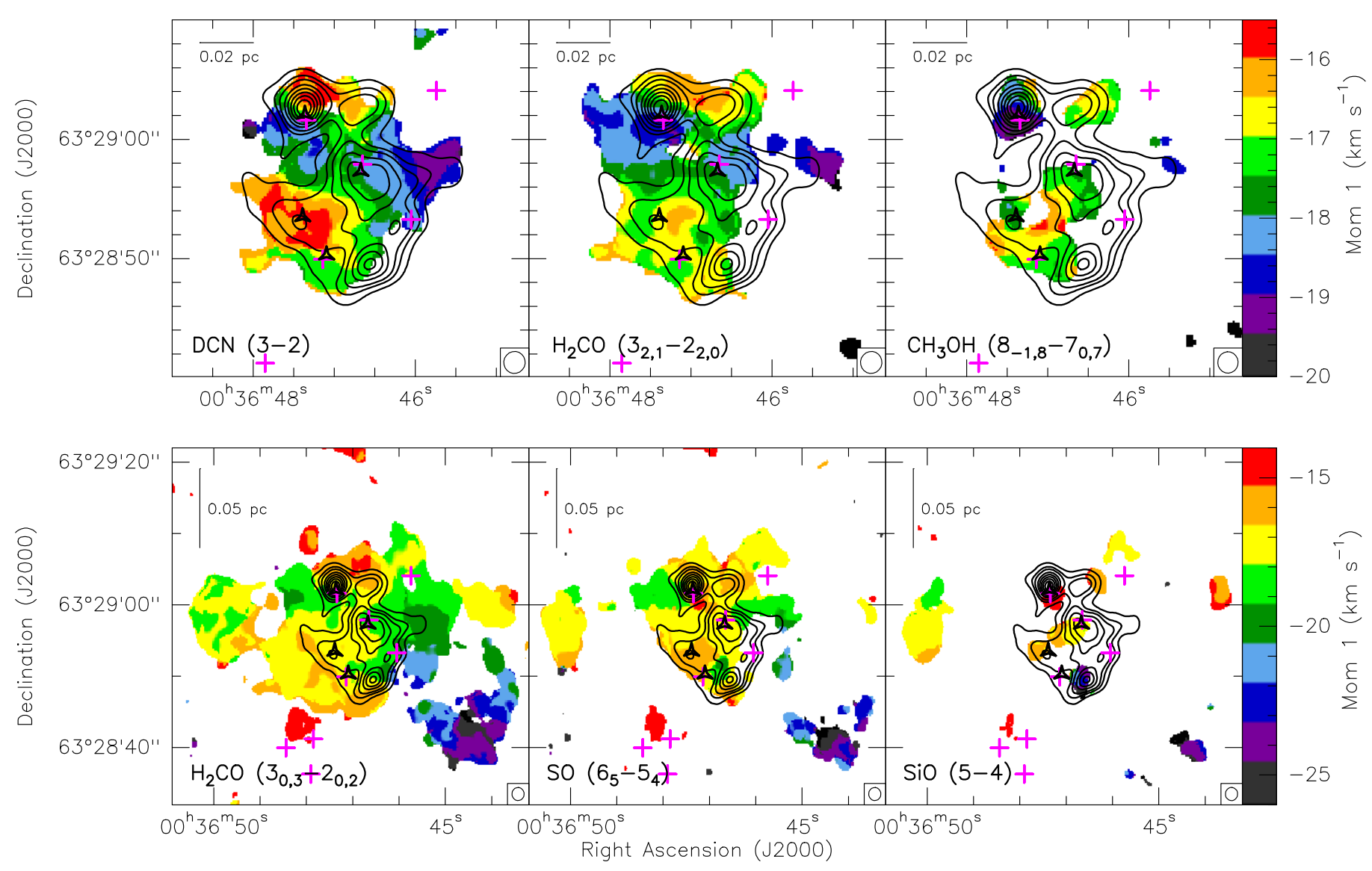

Fig. 4. Color scale image of the intensity-weighted average velocity (i.e., moment 1) maps of selected molecular line tracers overlapped with the contour map of the 1.3-mm dust continuum emission. Pink crosses and black triangles indicate infrared (Quanz et al. 2007) and radio (Anglada et al. 1994) sources, respectively. The synthesized beam of each molecule is located at the bottom-right corner.

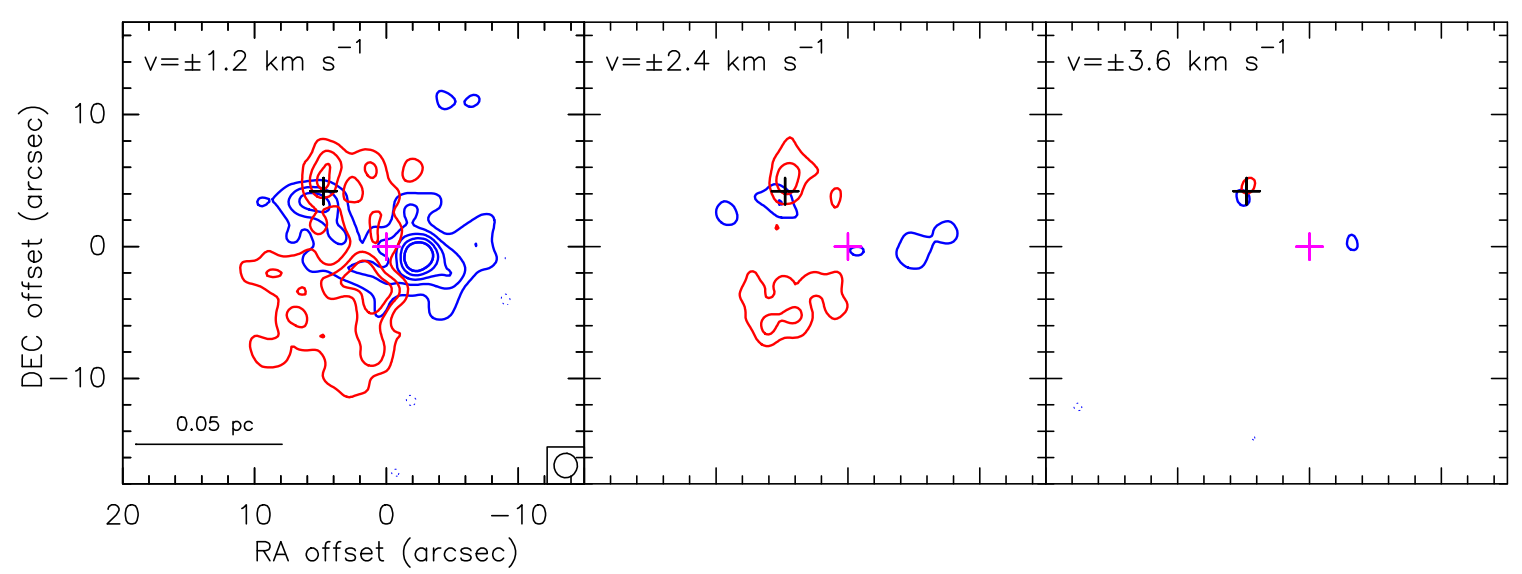

Fig. 5. Each panel shows the blue- and red-shifted emission of DCN (3-2) at $\pm 1.2, \pm 2.4$, and $\pm 3.6 \mathrm{kms}^{-1}$ from the systemic velocity $-17.4 \mathrm{~km} \mathrm{~s} \mathrm{~s}^{-1}$. Contours are $-4,4,8,12, \ldots, 20,30,40,50$ times the rms noise level of the map, $0.03 \mathrm{Jy} \mathrm{beam}^{-1}$. Black and pink crosses are IRAS $0038+6312$ and RNO1C sources, respectively. The synthesized beam located at the bottom-right corner of the first panel is $1^{\prime \prime} 85 \times 1^{\prime \prime} 77, \mathrm{PA}=-6^{\circ}$.

component of velocity gradient which has approximately the same orientation with the velocity gradient on $\sim 0.3-0.5 \mathrm{pc}$ scales. It is therefore natural to consider that our observations of these dense and/or warm gas tracers reveal the innermost part of the coherent gas accretion flow. Umemoto et al. (1999) interpreted this large-scale velocity gradient as rotational motions, due to the fact that it is perpendicular to the direction of the resolved $\mathrm{CO}$ outflow. Alternatively, it remains possible to coherently interpret the large-scale $(\sim 0.1-0.5 \mathrm{pc})$ velocity gradient and the inverse $\mathrm{P}$ Cygni line profile as infall. The presence of a $\mathrm{CO}$ outflow does not require a rotating disk on scales of $\gtrsim 0.1 \mathrm{pc}$. The velocities we detect from DCN (3-2) and $\mathrm{H}_{2} \mathrm{CO} 3(0,3)-2(0,2)$ at $\sim 12^{\prime \prime}(0.056 \mathrm{pc})$ from the center of SMA3 are $\sim 2 \mathrm{~km} \mathrm{~s}^{-1}$. The minimum required mass to gravitationally bind this motion is $\sim 25 M_{\odot}$, which is consistent with the lower limit of the gas and dust mass we estimated based on the $1.3-\mathrm{mm}$ dust continuum emission $\left(22 M_{\odot}\right.$; see Sect. 3.1).

In this context, the reversed velocity gradient on smaller spatial scales around IRAS $00338+6312$ was unexpected. In Fig. 10, we present the position-velocity (PV) diagrams made 


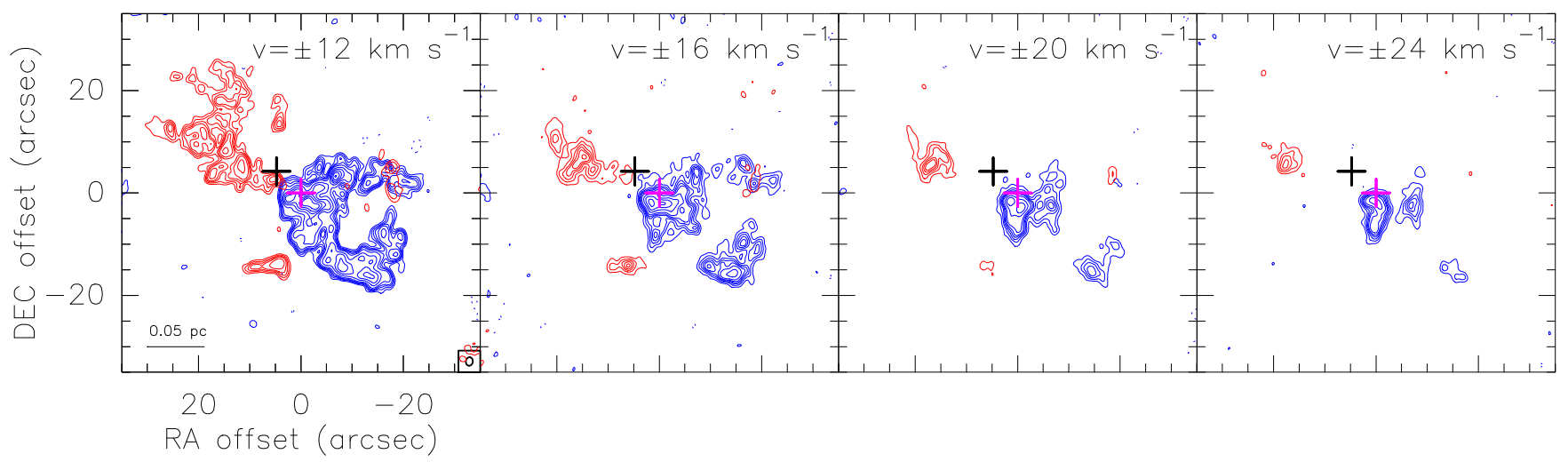

Fig. 6. CO (2-1) high-velocity emission. Each panel shows the red- and blue-shifted emission at $\pm 12, \pm 16, \pm 20$ and $\pm 24 \mathrm{~km} \mathrm{~s}^{-1}$ from the systemic velocity $-17.4 \mathrm{~km} \mathrm{~s}^{-1}$. Contour levels are $-6,6,9, \ldots, 15,20,30,40, \ldots, 70$ and $-3,3,6,9, \ldots, 15,20,30,40,50$ times the rms level $0.04 \mathrm{Jy} \mathrm{beam}^{-1}$ for the first and the rest of the panels, respectively. Black and pink crosses are IRAS 0038+6312 (also VLA 3) and RNO1C sources, respectively. The synthesized beam, located at bottom right of the first panel, is $1^{\prime \prime} 66 \times 1^{\prime \prime} 38, \mathrm{PA}=-8^{\circ}$. A scale bar is located in the bottom-left corner.

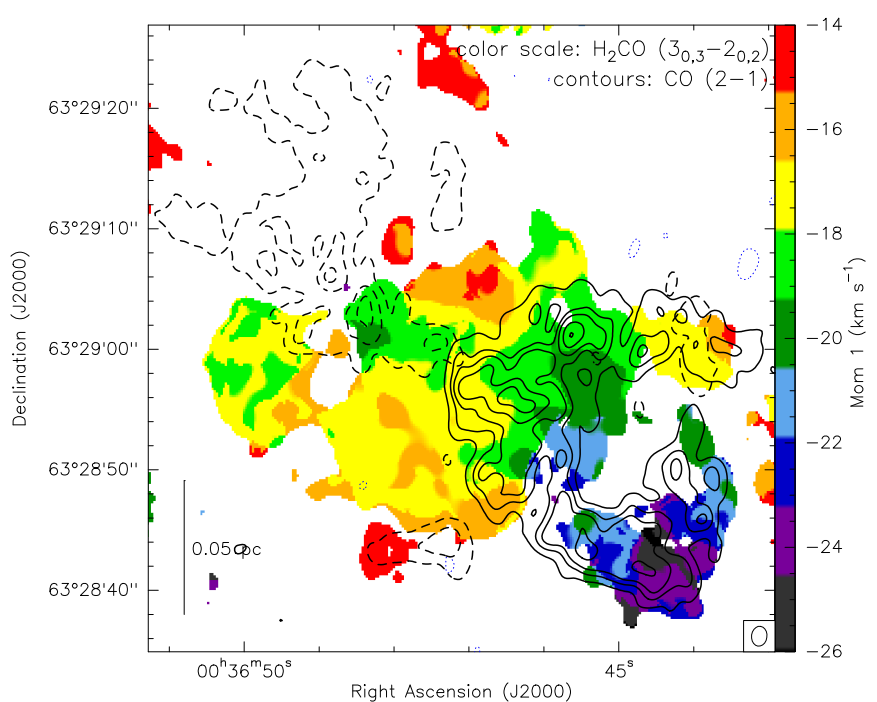

Fig. 7. Contours: CO (2-1) red- (dashed) and blue-shifted emission (solid) at $\pm 12 \mathrm{~km} \mathrm{~s}^{-1}$ from the systemic velocity $-17.4 \mathrm{~km} \mathrm{~s}^{-1}$. Contour levels are $-6,6,18,30,50,70$ times the rms level $0.04 \mathrm{Jy} \mathrm{beam}^{-1}$. The synthesized beam located in the bottom-right corner is $1^{\prime \prime} 66 \times$ $1^{\prime \prime} 38, \mathrm{PA}=-8^{\circ}$. Color scale: intensity-weighted averaged velocity (i.e., moment 1) map of $\mathrm{H}_{2} \mathrm{CO} 3(0,3)-2(0,2)$ emission. A scale bar is located in the bottom-left corner.

at the position angles of $140^{\circ}$ and $147^{\circ}$, centered at SMA3 and IRAS $00338+6312$, respectively. These two velocity gradients clearly have opposite directions. Interestingly, the PV diagram made at the position of IRAS $00338+6312$ resembles that of a rotating disk, while the other PV diagram does not. From the IRAS $0038+6312$ velocity gradient, assuming Keplerian motion $(v=\sqrt{G M / R}$, where $v$ is the velocity, $G$ is the gravitational constant, $M$ is the mass, and $R$ is the radius) and using a radius of $2.5^{\prime \prime}$ and $v=1.25 \mathrm{~km} \mathrm{~s}^{-1}$, we estimated a mass for IRAS $0038+6312$ of $4 M_{\odot}$. We note that the derived mass here is a lower limit as a possible inclination of the disk is not taken into account. The mass obtained is comparable to the $\sim 6 M_{\odot}$ obtained by Yang et al. (1991) using the mass-luminosity relation of $\left(L / L_{\odot}\right)=\left(M / M_{\odot}\right)^{4}$.

If we consider that the small-scale velocity gradient around IRAS $00338+6312$ is indeed due to rotational motion, then the reversion of the observed velocity gradients may be coherently interpreted as a filamentary large-scale inflow converging

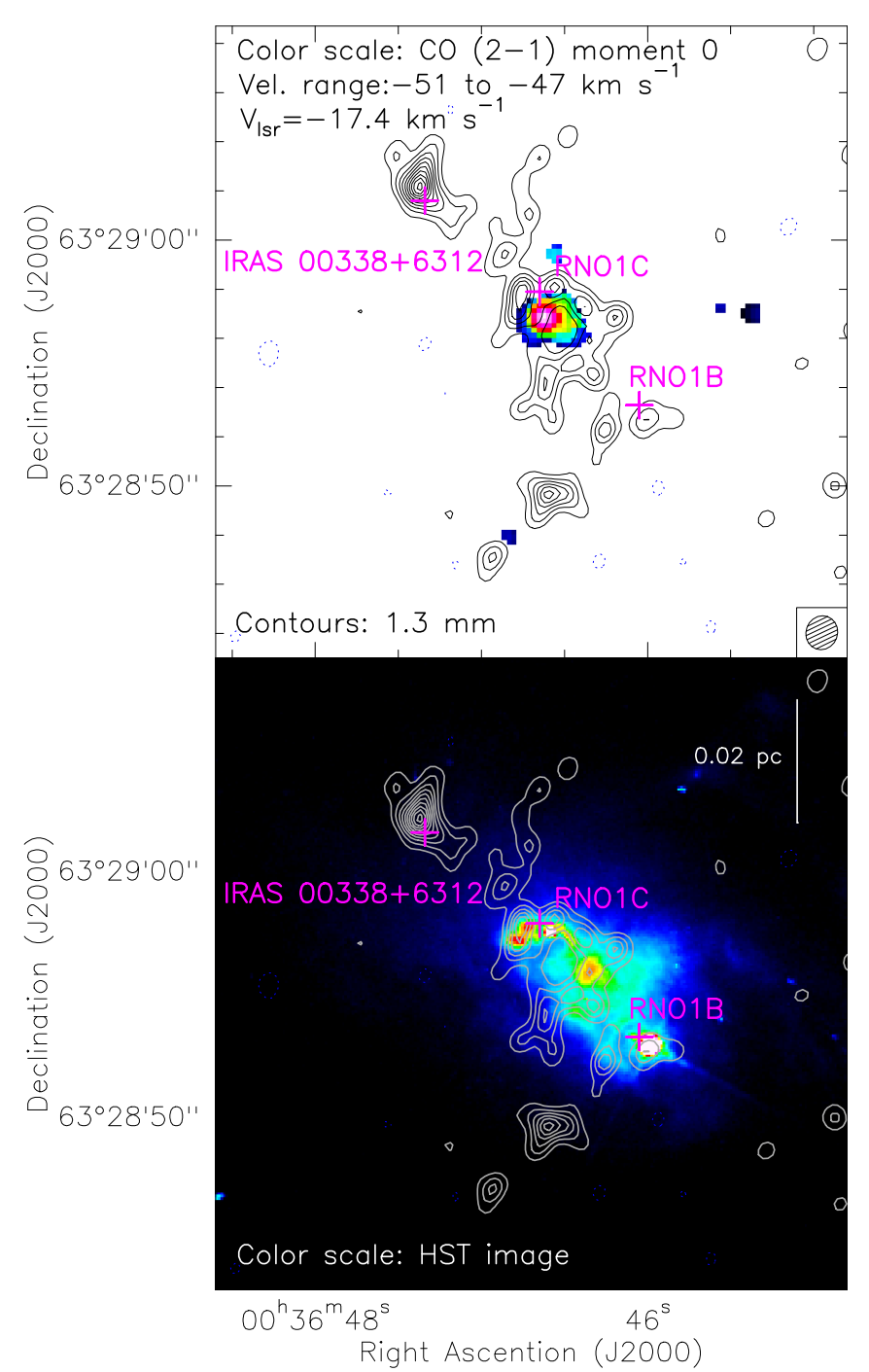

Fig. 8. Upper panel: $\mathrm{CO}(2-1)$ highest-velocity emission. Color scale: moment 0 (i.e., integrated intensity) map. Velocity range from -51 to $-47 \mathrm{~km} \mathrm{~s}^{-1}$. The synthesized beam located at the bottom right corner is $1^{\prime \prime} 39 \times 1^{\prime \prime} 27$, PA $=-19^{\circ}$. Contours: $1.3-\mathrm{mm}$ dust continuum emission. Pink crosses indicate infrared sources (Quanz et al. 2007). Lower panel: color scale - Hubble Space Telescope image. WFPC2 instrument, F814W filter. 

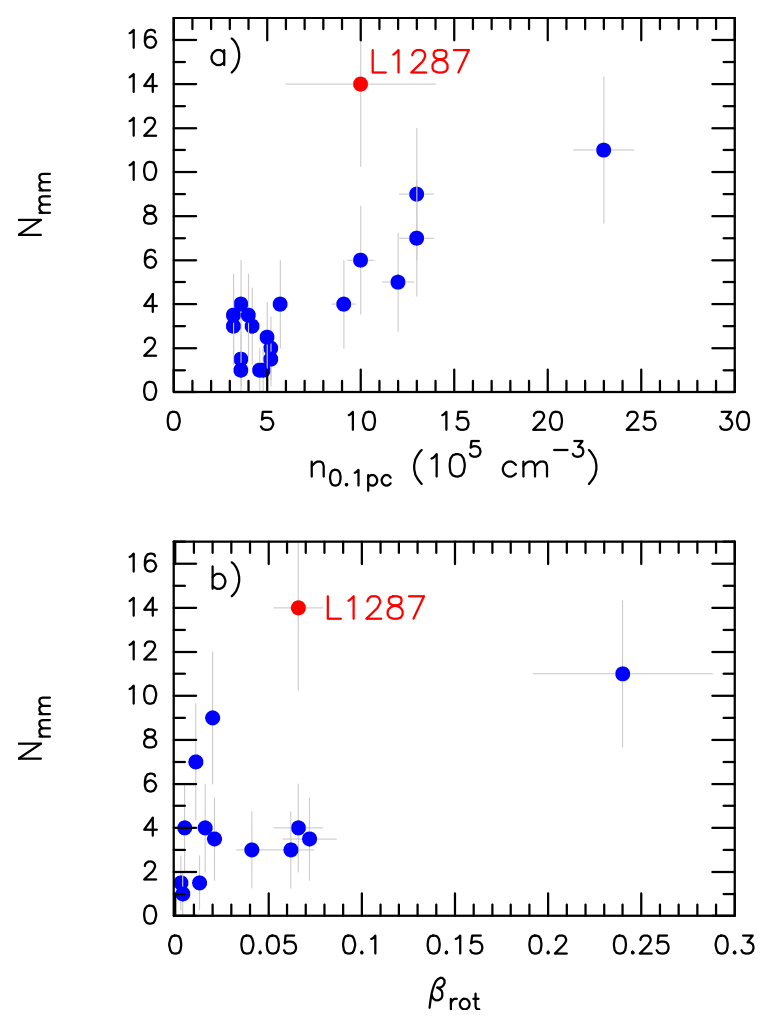

Fig. 9. Upper panel: fragmentation level $\left(N_{\mathrm{mm}}\right)$ vs. density averaged within a region of $0.1 \mathrm{pc}$ of diameter after Palau et al. $(2014,2015)$. Bottom panel: fragmentation level vs. rotational-to-gravitational energy after Palau et al. (2014). The location of L1287 in these plots is marked with a red symbol.

towards a circumstellar rotating disk, which may be further associated with the large-scale bipolar CO outflow (Sect. 3.2). The schematic diagram presented in Fig. 11 helps to illustrate this scenario. In the top panel of the figure, we present the blue- and red-shifted integrated DCN map showing the two low-velocity bipolar structures with the reversed velocity gradients. In the bottom panel of the figure, we present our favored interpretation for the reversed velocity gradients. Taking into account the considerations given above, the reversed velocity gradients could be interpreted as converging flows towards the IRAS $00338+6312$ source. The overall picture of forming a dense YSO cluster in this region from accreting dense gas filaments may therefore be analogous to those OB cluster-forming regions introduced in Sect. 1.

However, we have to point out that, observationally, it has been challenging to robustly distinguish rotational motion from infall, and is harder given the limited spectral resolution of our present observations. While our present interpretation with converging flow is partly motivated by the inverse P Cygni line profile on scales of $\gtrsim 0.1 \mathrm{pc}$ reported by Pirogov et al. (2016), we cannot yet rule out that our DCN data simply trace independent rotations around two centers, on smaller spatial scales. Future observations of molecular lines with better spectral resolution and higher sensitivity are still required to test our present interpretation.

\subsection{Powering source of the northeast-southwest molecular outflow}

The powering source of the reported northeast-southwest bipolar outflow in L1287 has been a matter of debate in the past, as this is also related to how the accretion and outflows of YSOs are linked (e.g. Evans et al. 1994). Several powering sources have

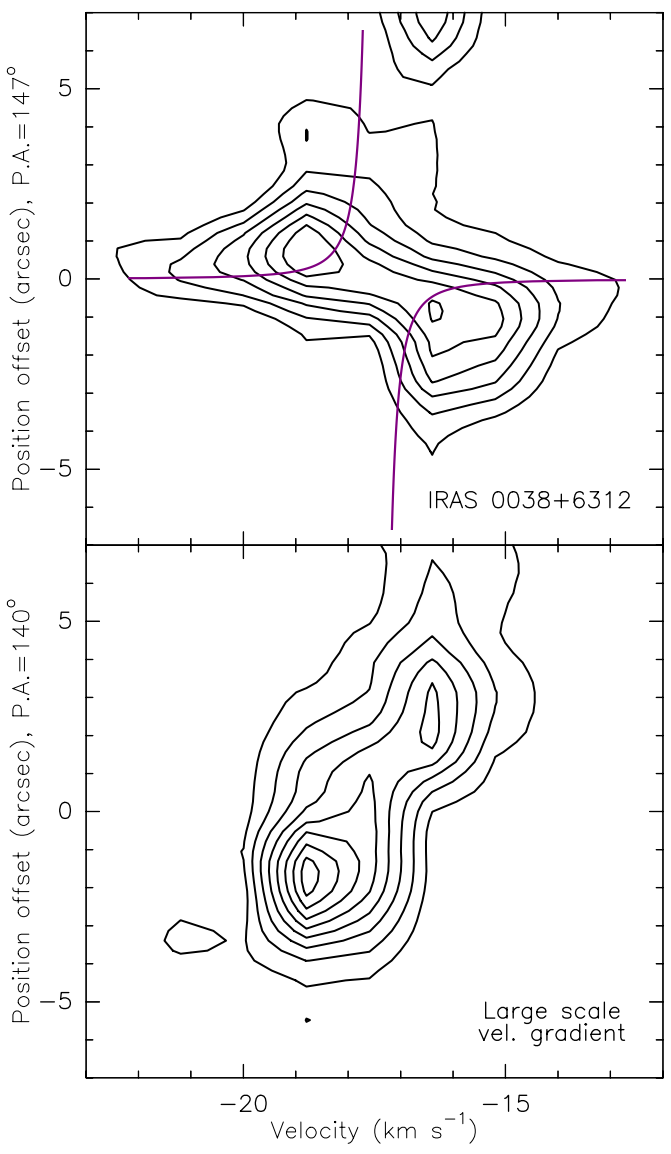

Fig. 10. DCN (3-2) position-velocity maps. Upper panel: emission along the velocity gradient at the IRAS $0038+6312$ source. Contours are $-3,3,5,7, \ldots, 13$ times the rms noise level of the map, $0.03 \mathrm{Jy}$ beam $^{-1}$. The purple lines indicate a keplerian velocity distribution. Lower panel: emission along the large velocity gradient passing through the center of the toroid traced by the dust continuum emission. Contours are $-3,3,6,9, \ldots, 24$ times the rms noise level of the map, $0.03 \mathrm{Jy}^{\text {beam }^{-1} \text {. }}$

been proposed, including RNO1B/1C (Staude \& Neckel 1991) and VLA 3 or IRAS $0038+6312$ (e.g. Anglada et al. 1994; Yang et al. 1995; Quanz et al. 2007).

By looking at the velocity range within $\pm 20 \mathrm{~km} \mathrm{~s}^{-1}$ with respect to the systemic velocity (Fig. 6), the facts that IRAS $00338+6312$ is located closer to the center of the bipolar $\mathrm{CO}$ outflow (Sect. 3.2), and that the velocity gradient of the dense gas around it appears perpendicular to the direction of the outflow, indicate that IRAS $00338+6312$ is a probable powering source and may be associated with a circumstellar (pseudo-)disk. However, at higher velocity (Fig. 6) the bright and blueshifted $\mathrm{CO}$ emission appears to be more closely associated with RNO $1 \mathrm{C}$. It may be possible that RNO $1 \mathrm{C}$ is also powering a monopolar $\mathrm{CO}$ outflow. The spatially compact nature of this outflow could be related to a recent accretion outburst event of this FU Orionis object. Supporting evidence may be the Hubble Space Telescope $\sim 8360 \AA$ (F814W filter) image shown in the lower panel of Fig. 8. At this wavelength the emission is associated with reflection or scattered light, and we would expect to detect it close to the blueshifted part of the emission, which is indeed the case. The brightest emission is clearly located towards the FU Orionis objects RNO 1B and RNO 1C. The emission also traces well the dust cavity structure seen with the high-angularresolution image of the SMA with peaks of emission at the SMA3c and SMA3f millimeter sources. 


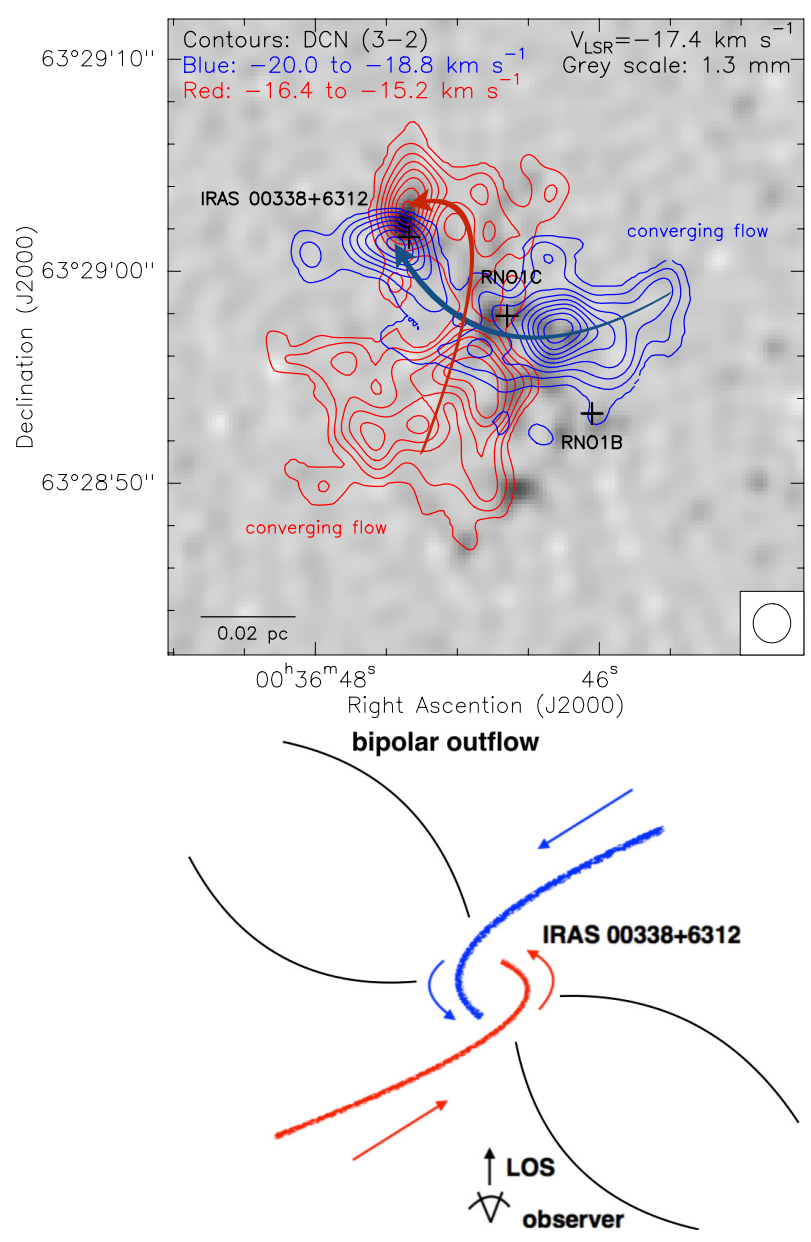

Fig. 11. Upper panel: contours correspond to the averaged emission from blue- and red-shifted channels of DCN (3-2) displaying the two low-velocity flow components. Contours are $6,9,12,15, \ldots, 30$ times the rms noise level of the map, $0.03 \mathrm{Jy} \mathrm{beam}^{-1}$. The synthesized beam is located at the bottom right corner. The grey scale corresponds to the $1.3 \mathrm{~mm}$ dust continuum emission. The arrows indicate the two possible converging flows of the region. Black crosses indicate the position of infrared sources IRAS $00338+6312$ and FU-Orionis RNO1B and RNO1C (Quanz et al. 2007). Lower panel: schematic model of the L1287 region. Converging flows + rotation dynamics. The blue and red lines indicate the $\sim 0.1 \mathrm{pc}$ scale molecular gas converging towards the center. We can observe a reversed velocity gradient projected towards our line of sight as we can see at the IRAS $00338+6312$ source.

\section{Conclusions}

We have performed $\sim 1^{\prime \prime}$ angular resolution SMA observations at $230 \mathrm{GHz}$ towards the inner $\sim 0.25$-pc cluster-forming region of the $\sim 10$-pc-scale filamentary dark cloud L1287. Our 1.3mm dust-continuum image resolves a $\sim 0.02$-pc-scale clumpy dense gas toroid located at the center, which is surrounded by elongated, 0.02-0.04-pc-scale arm-like dense gas structures and other spatially compact gas overdensities. The fragmentation level found in the region, as compared to previous studies, is very high, with up to 14 compact millimeter sources within a region of $0.1 \mathrm{pc}$ of diameter at a spatial resolution of $\sim 1000 \mathrm{AU}$.

In addition, our observations of the dense molecular gas tracers DCN (3-2) and $\mathrm{H}_{2} \mathrm{CO} 3(0,3)-2(0,2)$ resolved two components of velocity gradients: one has approximately the same direction as the velocity gradient traced by previous $\mathrm{H}^{13} \mathrm{CO}^{+}$ observations on $0.3-0.5 \mathrm{pc}$ scales; the other is seen on smaller spatial scales around the embedded YSO IRAS $00338+6312$, and presents a reversed direction. We cannot yet rule out that such incoherent motions are due to the influence of outflow feedback, or due to turbulent motions; and cannot yet rule out that we are seeing independent rotational motions around two centers. However, we find that these motions might be coherently interpreted as a filamentary, 0.1-0.5-pc-scale infalling gas flow, which is converging towards the rotating circumstellar disks on smaller spatial scales. The formation of the low-mass YSO cluster at the center of L1287 might be fed by the global gas inflow, which is analogous to some luminous OB clusterforming regions such as W49A, G10.6-0.4, G33.92+0.11, and NGC6334 V.

Acknowledgements. We thank the SMA staff for their support which makes these studies possible. C.J. acknowledges support from MINECO (Spain) BES-2012052481 grant. C.J., J.M.G. and G.B. acknowledge support from MICINN (Spain) AYA2014-57369-C3 and AYA2017-84390-C2-2-R grants. A.P. acknowledges financial support from UNAM and CONACyT, México. R.G.M. acknowledges support from UNAM-PAPIIT program IA102817.

\section{References}

Anglada, G., Rodriguez, L. F., Girart, J. M., Estalella, R., \& Torrelles, J. M. 1994, ApJ, 420, L91

Beltrán, M. T., Estalella, R., Anglada, G., Rodríguez, L. F., \& Torrelles, J. M. 2001, AJ, 121, 1556

Briggs, D. S. 1995, BAAS, 27, 1444

Corsaro, E., Lee, Y.-N., García, R. A., et al. 2017, Nat. Astron., 1, 0064

Di Francesco, J., Johnstone, D., Kirk, H., MacKenzie, T., \& Ledwosinska, E. 2008, ApJS, 175, 277

Estalella, R., Mauersberger, R., Torrelles, J. M., et al. 1993, ApJ, 419, 698

Evans, II, N. J., Balkum, S., Levreault, R. M., Hartmann, L., \& Kenyon, S. 1994, ApJ, 424, 793

Fehér, O., Kóspál, Á., Ábrahám, P., Hogerheijde, M. R., \& Brinch, C. 2017, A\&A, 607, A39

Galván-Madrid, R., Liu, H. B., Zhang, Z.-Y., et al. 2013, ApJ, 779, 121

Girart, J. M., Viti, S., Estalella, R., \& Williams, D. A. 2005, A\&A, 439, 601

Hartmann, L., \& Kenyon, S. J. 1985, ApJ, 299, 462

Herbig, G. H. 1977, ApJ, 217, 693

Hildebrand, R. H. 1983, QJRAS, 24, 267

Ho, P. T. P., Moran, J. M., \& Lo, K. Y. 2004, ApJ, 616, L1

Juárez, C., Girart, J. M., Zamora-Avilés, M., et al. 2017, ApJ, 844, 44

Kenyon, S. J., Hartmann, L., Gomez, M., Carr, J. S., \& Tokunaga, A. 1993, AJ, 105,1505

Keto, E. R., Ho, P. T. P., \& Haschick, A. D. 1987, ApJ, 318, 712

Keto, E. R., Lattanzio, J. C., \& Monaghan, J. J. 1991, ApJ, 383, 639

Lefloch, B., Cernicharo, J., Cabrit, S., \& Cesarsky, D. 2005, A\&A, 433, 217

Lin, Y., Liu, H. B., Li, D., et al. 2016, ApJ, 828, 32

Liu, H. B., Quintana-Lacaci, G., Wang, K., et al. 2012a, ApJ, 745, 61

Liu, H. B., Jiménez-Serra, I., Ho, P. T. P., et al. 2012b, ApJ, 756, 10

Liu, H. B., Galván-Madrid, R., Jiménez-Serra, I., et al. 2015, ApJ, 804, 37

Liu, H. B., Dunham, M. M., Pascucci, I., et al. 2018, A\&A, 612, A54

Mapelli, M. 2017, MNRAS, 467, 3255

McMuldroch, S., Blake, G. A., \& Sargent, A. I. 1995, AJ, 110, 354

Molinari, S., Swinyard, B., Bally, J., et al. 2010, A\&A, 518, L100

Myers, P. C. 2009, ApJ, 700, 1609

Ossenkopf, V., \& Henning, T. 1994, A\&A, 291, 943

Palau, A., Fuente, A., Girart, J. M., et al. 2013, ApJ, 762, 120

Palau, A., Estalella, R., Girart, J. M., et al. 2014, ApJ, 785, 42

Palau, A., Ballesteros-Paredes, J., Vázquez-Semadeni, E., et al. 2015, MNRAS, 453, 3785

Pirogov, L. E., Shul'ga, V. M., Zinchenko, I. I., et al. 2016, Astron. Rep., 60, 904 Quanz, S. P., Henning, T., Bouwman, J., Linz, H., \& Lahuis, F. 2007, ApJ, 658, 487

Rygl, K. L. J., Brunthaler, A., Reid, M. J., et al. 2010, A\&A, 511, A2

Sault, R. J., Teuben, P. J., \& Wright, M. C. H. 1995, in Astronomical Data Analysis Software and Systems IV, eds. R. A. Shaw, H. E. Payne, \& J. J. E. Hayes, ASP Conf. Ser., 77, 433

Snell, R. L., Dickman, R. L., \& Huang, Y.-L. 1990, ApJ, 352, 139

Staude, H. J., \& Neckel, T. 1991, A\&A, 244, L13

Umemoto, T., Saito, M., Yang, J., \& Hirano, N. 1999, in Star Formation 1999, ed. T. Nakamoto (Japan: Nobeyama Radio Observatory), 227

Yang, J., Umemoto, T., Iwata, T., \& Fukui, Y. 1991, ApJ, 373, 137

Yang, J., Ohashi, N., \& Fukui, Y. 1995, ApJ, 455, 175 


\section{Appendix A: Line identifications}

In this appendix we present a table with the list of detected transitions towards L1287 with the SMA (Table A.1).

Table A.1. Molecular lines detected with the SMA towards L1287.

\begin{tabular}{ccccc}
\hline \hline Molecule & Transition & $\begin{array}{c}\text { Frequency } \\
(\mathrm{GHz})\end{array}$ & $\begin{array}{c}E_{\mathrm{U}} \\
(\mathrm{K})\end{array}$ & $\begin{array}{c}\rho_{\text {crit }}^{a} \\
\left(\mathrm{~cm}^{-3}\right)\end{array}$ \\
\hline $\mathrm{CH}_{3} \mathrm{OH}$ & $5(1,4)-4(2,2)$ & 216.946 & 56 & $2.33 \times 10^{6}$ \\
$\mathrm{SiO}$ & $(5-4)$ & 217.105 & 31 & $3.25 \times 10^{8}$ \\
$\mathrm{DCN}$ & $(3-2)$ & 217.239 & 21 & - \\
$\mathrm{H}_{2} \mathrm{CO}$ & $3(0,3)-2(0,2)$ & 218.222 & 21 & $2.56 \times 10^{6}$ \\
$\mathrm{H}_{2} \mathrm{CO}$ & $3(2,2)-2(2,1)$ & 218.476 & 68 & $2.96 \times 10^{6}$ \\
$\mathrm{H}_{2} \mathrm{CO}$ & $3(2,1)-2(2,0)$ & 218.760 & 68 & $3.36 \times 10^{6}$ \\
$\mathrm{OCS}$ & $(18-17)$ & 218.903 & 100 & $4.28 \times 10^{5}$ \\
$\mathrm{C}^{18} \mathrm{O}$ & $(2-1)$ & 219.560 & 16 & $9.33 \times 10^{3}$ \\
$\mathrm{HNCO}$ & $10(0,10)-9(0,9)$ & 219.798 & 58 & - \\
$\mathrm{SO}$ & $6(5)-5(4)$ & 219.949 & 35 & - \\
${ }_{13} \mathrm{CO}$ & $(2-1)$ & 220.399 & 16 & $9.38 \times 10^{3}$ \\
$\mathrm{DNC}$ & $(3-2)$ & 228.910 & 22 & - \\
$\mathrm{CH}_{3} \mathrm{OH}$ & $8(-1,8)-7(0,7)$ & 229.759 & 89 & $2.62 \times 10^{7}$ \\
$\mathrm{CH}_{3} \mathrm{OH}$ & $3(-2,2)-4(-1,4)$ & 230.027 & 40 & $7.05 \times 10^{6}$ \\
$\mathrm{CO}$ & $(2-1)$ & 230.538 & 17 & $1.07 \times 10^{4}$ \\
\hline
\end{tabular}

Notes. ${ }^{(a)} \rho_{\text {crit }}=A_{\mathrm{ul}} / \gamma$, where $A_{\mathrm{ul}}$ is the Einstein spontaneous emission coefficient and $\gamma=\sigma\langle\mathrm{v}\rangle$ is the collisional rate where $\sigma$ is the cross section of the collision for each transition and $\langle\mathrm{v}\rangle \approx$ $\left(3 \mathrm{kT} \mathrm{m}^{-1}\right)^{1 / 2}$ is the average velocity of the collisional particles. As $\mathrm{H}_{2}$ is the most abundant molecule it is used as the dominant collisional particle. The Einstein spontaneous emission coefficients are taken from LAMDA (http://home.strw.leidenuniv. $\mathrm{nl} /$ moldata) and splatalogue.net databases and $\gamma$ values are taken from LAMDA database. We used $T=20 \mathrm{~K}$ (as $22 \mathrm{~K}$ is not available in the tabulated data). 